\title{
Superficial Femoral Artery Calcification Is a Novel Risk Factor of Microvascular Complications in T2DM Patients
}

\author{
Jing Tian ${ }^{1} \cdot$ Wanbing $\mathrm{He}^{2} \cdot$ Jingwei Gao ${ }^{2} \cdot \mathrm{Li} \mathrm{Yan}^{3} \cdot$ Ming Liang ${ }^{1} \cdot$ Wenyue Zhang ${ }^{1} \cdot \mathrm{Xiaolin}_{\mathrm{Xu}}{ }^{1} \cdot$ Baoming Luo $^{1}$
}

Received: 10 August 2019 / Accepted: 30 November 2019 / Published online: 13 January 2020

(c) The Author(s) 2020

\begin{abstract}
Microvascular complications are prevalent in patients with type 2 diabetes mellitus (T2DM), resulting in increased risk of cardiovascular mortality. However, it is unclear whether above-knee artery calcification relates to microvascular complications. This study was aimed to investigate the role of calcification in superficial femoral arteries (SFA), the major above-knee artery, compared with anterior tibial arteries (ATA) and posterior tibial arteries (PTA), in T2DM-related microvascular complications and explore its risk factors. A single-center and observational study involving 359 T2DM patients was conducted. Clinical and laboratory data were collected. SFA calcification was evaluated by ultrasonography. Compared with ATA and PTA calcification, operating characteristics curve analysis showed that SFA calcification was the strongest predictor $(63.1 \%$ sensitivity and $69.2 \%$ specificity) for T2DM-related microvascular complications (diabetic neuropathy, diabetic nephropathy and diabetic retinopathy). With the severity of SFA calcification increased, age, duration of T2DM, and SBP were significantly elevated, but triglyceride and glucose index and estimated glomerular filtration rate (eGFR) were significantly reduced (all $P<0.05$ ). Multivariate logistic analysis showed that eGFR (OR 0.953; 95\% CI 0.931-0.976; $P<0.001$ ) was an independent risk factor of SFA calcification, especially in young patients with HbA1c $>7.0$. We identified SFA calcification as a good predictor of microvascular complications in T2DM patients. Reduced eGFR was significantly associated with increased SFA calcification prevalence, especially in young T2DM patients with bad controlled hyperglycemia.
\end{abstract}

Keywords Type 2 diabetes mellitus $\cdot$ Superficial femoral artery calcification · Microvascular complications · Estimated glomerular filtration rate $\cdot$ Hyperglycemia

\section{Abbreviations}

ATA Anterior tibial arteries

BMI Body mass index

Jing Tian, Wanbing He and Jingwei Gao have contributed equally to this work.

Xiaolin Xu

xuxiaolin@mail.sysu.edu.cn

Baoming Luo

luobm@mail.sysu.edu.cn

1 Department of Ultrasound, Guangdong Provincial Key Laboratory of Malignant Tumor Epigenetics and Gene Regulation, Medical Research Center, Sun Yat-Sen Memorial Hospital of Sun Yat-Sen University, 107 West Yanjiang Road, Guangzhou 510120, China

2 Department of Cardiology, Sun Yat-Sen Memorial Hospital of Sun Yat-Sen University, Guangzhou, China

3 Department of Endocrinology, Sun Yat-Sen Memorial Hospital of Sun Yat-Sen University, Guangzhou 510120, China
DBP Diastolic blood pressure

eGFR Estimated glomerular filtration rate

FPG Fasting plasma glucose

HbA1c Hemoglobin A1c

HDL-C High-density lipoprotein cholesterol

LDL-C Low-density lipoprotein cholesterol

MAC Medial arterial calcification

PAD Peripheral arterial disease

PTA Posterior tibial arteries

ROC Receiver-operating characteristics curve

SBP Systolic blood pressure

SFA Superficial femoral arteries

TC Total cholesterol

T2DM Type 2 diabetes mellitus

TG Triglycerides

TyG index Triglyceride and glucose index

WC Waist circumference 


\section{Background}

Microvascular complications (neuropathy, nephropathy and retinopathy) affect hundreds of millions of patients with type 2 diabetes mellitus (T2DM) [1-3]. The progression of these complications can lead to loss of visual, renal, and neurologic functions, impaired mobility and cognition, poor quality of life, limitations for employment and productivity, and increased costs for the patient and society $[4,5]$. If left uncontrolled or untreated, they lead to irreversible damage and even death. Thus, it is of great importance to investigate the risk factors and carry early intervention [6].

Arterial calcification in the lower extremity, particularly in the setting of T2DM, doubles cardiovascular mortality and quadruples the risk of amputation [7]. It is classified as two forms: intimal calcification is a marker of atherosclerotic disease and is associated with arterial stenosis; while T2DM patients usually have medial arterial calcification (MAC) [8]. MAC is a condition that leads to stiffening of the elastic layer of the arterial wall, resulting in a series of cardiovascular events [9]. Ultrasonography is widely used to assess arterial intimal abnormalities such as intima-medial thickness [10] and endothelial dysfunction [11]. Compared with conventional radiography, ultrasound can directly aid visualization of the arterial lumen of the peripheral arterial tree and has its advantages in detecting MAC accurately [12]. Previous studies mostly focused on the association between MAC in lower limb arteries and various diabetic macrovascular complications including peripheral arterial diseases (PAD) [13, 14]. Lower limb arteries mainly consist of above-knee arteries [superficial femoral arteries (SFA)], and below-knee arteries [anterior tibial arteries (ATA) and posterior tibial arteries (PTA)]. However, it remains a debate on whether below- or above-knee calcification plays a role in T2DM-related microvascular complications. Guzman RJ et al. showed that tibial artery calcification was associated with foot ulcer in T2DM [15]. By contrast, the results from a recent study indicated an association between SFA calcification and T2DM-related microvascular complications [16]. These studies were limited for two reasons. On one hand, they used computed tomography measurement, which may underestimate MAC severity; on the other hand, they just measured one segment of lower limb arteries and did not explore the related risk factors either.

Thus, in this present study, we improved these flaws and adapted a sensitive method (ultrasonography) to evaluate MAC in the whole lower limb artery branches (SFA, ATA and PTA) of T2DM patients. We aimed to assess which lower limb artery segment calcification was associated with diabetes-related complications and to evaluate its risk factors.

\section{Methods}

\section{Study Participants}

This was a retrospective, single-center study consisting of T2DM patients admitted in our unit between March 2015 and December 2017. The demographic, biomedical and US profiles were extracted from medical database. The main inclusion criteria were based on the T2DM diagnostic guideline on the 1999 World Health Organization [17]. The main exclusion criteria were (1) type 1 diabetes mellitus; (2) recent infection inflammatory disorders, serious cardiovascular diseases, renal dysfunction or hepatic diseases; (3) recent receiving treatments of several agents affecting mineral metabolism such as steroid hormones and anti-osteoporosis drugs within three months; (4) malignancy or disability to complete required measurement. This study protocol conformed to the ethical guidelines of the Declaration of Helsinki as reflected in a priori approval by the Ethics Committee of Sun Yat-sen University.

\section{Measurement of Blood Pressure and Body Mass Index (BMI)}

Systolic blood pressure (SBP) and diastolic blood pressure (DBP) were measured three times using digital sphygmomanometers (OMRON Healthcare, Hoofddorp, The Netherlands) and the mean values of measurements were used as SBP and DBP in the analysis. BMI was calculated by weight $/$ height ${ }^{2}\left(\mathrm{~kg} / \mathrm{m}^{2}\right)$.

\section{Biochemical Measurements}

Blood samples were collected after an overnight fast for at least $8 \mathrm{~h}$. Fasting plasma glucose (FPG), hemoglobin A1c (HbA1c), serum total cholesterol (TC), triglycerides (TG), high-density lipoprotein cholesterol (HDL-C) and low-density lipoprotein cholesterol (LDL-C), creatinine, calcium and phosphorus were measured on a standardized and certified TBA-120 auto-analyzer (Toshiba Medical Systems, Japan) in the central laboratory of our unit. Notably, the triglyceride and glucose $(\mathrm{TyG})$ index $(\mathrm{Ln}[\mathrm{TG}(\mathrm{mg} / \mathrm{mL}) \times$ FPG $(\mathrm{mg} /$ $\mathrm{mL}$ )/2]) and estimated glomerular filtration rate (eGFR) were calculated with established formulas: $141 \times \min$ $(\mathrm{Scr} / \kappa, 1) \alpha \times \max (\mathrm{Scr} / \kappa, 1)-1.209 \times 0.993^{\mathrm{Age}} \times 1.018[\mathrm{if}$ female]_1.159[if black], where $\kappa$ is 0.7 for females and 0.9 for males, $\alpha$ is -0.329 for females and -0.411 for males, min indicates the minimum of $\mathrm{Scr} / \kappa$ or 1 , and max indicates the maximum of $\mathrm{Scr} / \kappa$ or 1 [18]. 


\section{Ultrasonography Detection of MAC}

All the T2DM patients underwent ultrasonography examination of bilateral superficial femoral arteries for the presence of MAC. An ultrasonography unit (LOGIQ E9, GE, USA) was used with an L5-12 MHz or L9 MHz transducer. The examinations were performed and interpreted by two sonographers, who were blinded to the clinical data. The SFA, ATA and PTA of both thighs were scanned in the crosssectional plane from their origins at the groin to their distal parts at the entrance of the adductor canal to have an overall view of the blood vessels (Fig. 1). The MAC was assigned a score from 0 to 4 according to severity of the calcification within a 4-cm scanned area (which was usually the approximate size of the ultrasonography transducer). The definitions of unilateral artery were as follows: score 0 , no MAC; score 1 , linear MAC with a length of less than $1 \mathrm{~cm}$; score 2, linear MAC with a length between 1 and $2 \mathrm{~cm}$; score 3 , more
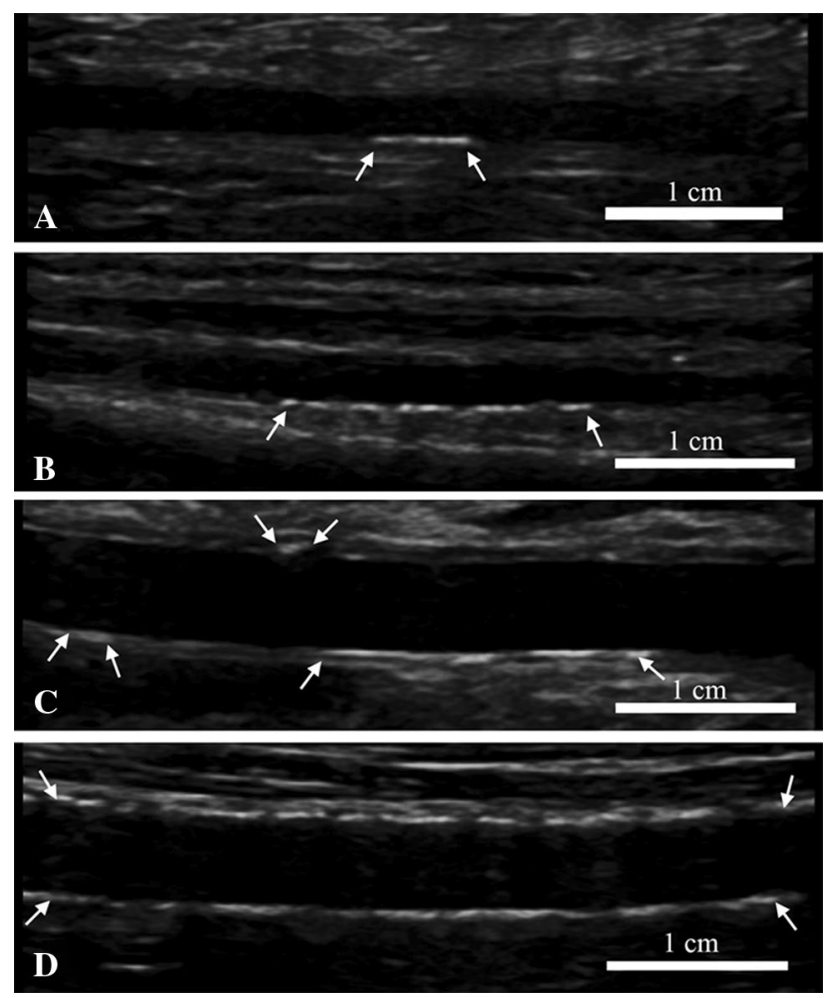

Fig. 1 Representative ultrasound images from T2DM population with different MAC severities in the lower limb arteries. a MAC with score 1 (linear calcification length of PTA is less than $1 \mathrm{~cm}$ in this scanning area). b MAC with score 2 (linear calcification length of ATA occupies approximately $1-2 \mathrm{~cm}$ in this scanning area). c MAC with score 3 (more extensive non-stenotic calcifications of PTA with approximately $2-3 \mathrm{~cm}$ in this scanning area). d MAC with score 4 (diffuse linear calcification of SFA with extent more than $3 \mathrm{~cm}$ in this scanning area). The white arrows indicate MAC. ATA, anterior tibial arteries; MAC, medial arterial calcification; PTA, posterior tibial arteries; SFA, superficial femoral arteries; T2DM, type 2 diabetes extensive non-stenotic MAC with a length between 2 and $3 \mathrm{~cm}$; and score 4, diffuse linear MAC with a length more than $3 \mathrm{~cm}$ (Fig. 1) [12].

\section{Clinical Assessment of Diabetic Complications}

The included patients underwent comprehensive clinical evaluations. The definitions of various diabetic complications were consistent with recent guidelines [19-21]. The definition of diabetic neuropathy was as follows: if T2DM patients had clinical symptoms like pain, numbness, abnormal sensation, etc., accompanied with any one abnormality in examinations (ankle reflex, acupuncture pain, vibration, pressure and temperature sensation); or in the absence of clinical symptoms, 2 out of 5 tests were abnormal [19]. Diabetic nephropathy was defined as overt albuminuria (urinary albumin excretion rate $>300 \mathrm{mg} / 24 \mathrm{~h}$ ) and eGFR $<60 \mathrm{~mL} /$ min per $1.73 \mathrm{~m}^{2}$ (no end-stage renal disease or kidney transplantation) in T2DM, without any clinical or laboratory evidence of other kidney diseases [20]. Diabetic retinopathy was defined as the presence of dot and blot hemorrhages, hard exudates, cotton wool spots, neovascularization, laser scars, or a history of vitrectomy in T2DM [21].

\section{Statistical Analysis}

Continuous variables with a normal distribution were reported as mean \pm standard deviation; skewed data as median (25-75th quartiles). Categorical variables were presented as numbers (percentages). Baseline variables among patients with different severity of SFA calcification groups were compared using variance or Kruskal-Wallis test followed by a LSD comparison test or Pearson chi-square according to the data types. Receiver-operating characteristics curve (ROC) analysis was used to quantify the assessing value of different lower limb artery calcification for diabetic complications. Univariate and multivariate logistic analysis were used to investigate the risk factors for SFA calcification, as well as in further subgroup analysis. Data were analyzed with SPSS version 20 (SPSS, Inc, Chicago, IL), and two-sided $P$ values $<0.05$ were considered statistically significant.

\section{Results}

\section{Characteristics of Recruited T2DM Patients}

A total of 359 consecutive patients with T2DM were finally included in this study. Baseline characteristics of all the T2DM patients were summarized in Table 1. The mean age was 58.6 \pm 11.6 years and $162(45.1 \%)$ T2DM patients were male. The mean SBP and DBP 
Table 1 Baseline characteristics of included T2DM patients

\begin{tabular}{ll}
\hline Variables & $\begin{array}{l}\text { Total enrolled } \\
\text { patients } \\
(N=359)\end{array}$ \\
\hline Age (years) & $58.6 \pm 11.6$ \\
Sex $(\mathrm{M} / \mathrm{F})$ & $162 / 197$ \\
Smoking $[N(\%)]$ & $117(32.6)$ \\
Duration of T2DM (years) & $5.0(0-12.0)$ \\
SBP (mmHg) & $134.8 \pm 21.3$ \\
DBP (mmHg) & $78.5 \pm 11.6$ \\
BMI (m $/ \mathrm{kg})$ & $24.0 \pm 3.1$ \\
WC (cm) & $88.4 \pm 8.8$ \\
FPG (mmol/L) & $8.6 \pm 3.4$ \\
HbAc1 $(\%)$ & $9.6 \pm 2.6$ \\
TG (mmol/L) & $1.52(1.10-2.28)$ \\
TC (mmol/L) & $5.00 \pm 1.60$ \\
HDL-C (mmol/L) & $1.09 \pm 0.34$ \\
LDL-C (mmol/L) & $3.14 \pm 1.03$ \\
Calcium (mmol/L) & $2.24 \pm 0.12$ \\
Phosphorus (mmol/L) & $1.17 \pm 0.21$ \\
Creatinine (mmol/L) & $70.0(81.1-99.0)$ \\
eGFR (mL/min per 1.73 m $\left.{ }^{2}\right)$ & $75.83 \pm 16.13$ \\
TyG index & $9.27 \pm 0.75$ \\
SFA calcification $[N(\%)]$ & $210(58.5)$ \\
ATA calcification $[N(\%)]$ & $140(39.0)$ \\
PTA calcification $[N(\%)]$ & $135(37.6)$ \\
Diabetic neuropathy $[N(\%)]$ & $227(63.2)$ \\
Diabetic nephropathy $[N(\%)]$ & $105(29.2)$ \\
Diabetic retinopathy $[N(\%)]$ & $103(28.7)$ \\
Metformin $[N(\%)]$ & $155(43.2)$ \\
Other hypoglycemic agents $[N(\%)]$ & $190(52.9)$ \\
Insulin $[N(\%)]$ & $125(34.8)$ \\
\hline & \\
&
\end{tabular}

Values presented as mean $\pm \mathrm{SD}$ or the median $\left(25^{-} 75\right.$ th quartiles)

$A T A$ anterior tibial arteries, $B M I$ body mass index, $D B P$ diastolic blood pressure, $e G F R$ estimated glomerular filtration rate, $F P G$ fasting plasma glucose, $H b A l c$ hemoglobin A1c, $H D L-C$ high-density lipoprotein cholesterol, $L D L-C$ low-density lipoprotein cholesterol, $P T A$ posterior tibial arteries, $S B P$ systolic blood pressure, $S F A$ superficial femoral arteries, $T 2 D M$ type 2 diabetes mellitus, $T C$ total cholesterol, $T y G$ index triglyceride and glucose index, $T G$ triglycerides, $W C$ waist circumference

were $134.8 \pm 21.3$ and $78.5 \pm 11.6 \mathrm{mmHg}$, respectively. The median duration of T2DM was $5.0(0-12.0)$ years. Moreover, the mean serum calcium, phosphorus, eGFR and TyG index in these patients were $2.24 \pm 0.12 \mathrm{mmol} / \mathrm{L}$, $1.17 \pm 0.21 \mathrm{mmol} / \mathrm{L}, 75.83 \pm 16.13 \mathrm{~mL} / \mathrm{min}$ per $1.73 \mathrm{~m}^{2}$ and $9.27 \pm 0.75$, respectively. The prevalence of calcification in different lower limb artery branches were: SFA calcification 210 (58.5\%), ATA calcification 140 (39.0\%) and PTA calcification 135 (37.6\%). As regard to diabetic microvascular complications, 227 (63.2\%) T2DM patients

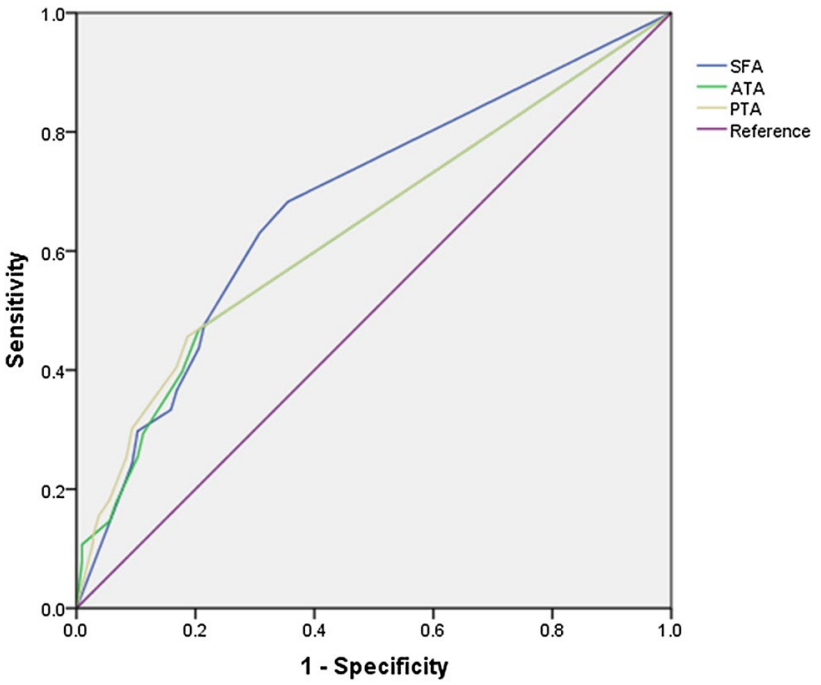

Fig. 2 Receiver-operating characteristic curve for SFA, ATA and PTA calcification in assessing T2DM-related microvascular complications, respectively. ATA anterior tibial arteries, PTA posterior tibial arteries, $S F A$ superficial femoral arteries, $T 2 D M$ type 2 diabetes

had diabetic neuropathy, 105 (29.2\%) T2DM patients had diabetic nephropathy, and 103 (28.7\%) T2DM patients had diabetic retinopathy.

\section{SFA Calcification Is a Good Marker for Predicting Diabetic Complications}

To explore the roles of different lower limb artery root calcification for predicting diabetic microvascular complications, we performed ROC curve analysis. As shown in Fig. 2, compared with ATA, PTA calcification, the area under the curve for SFA calcification was the largest [SFA vs. ATA vs. PTA: $0.679(0.619,0.739)$ vs. $0.636(0.577,0.696)$ vs. 0.641 (0.582, 0.700); all $P<0.001]$. SFA calcification with a threshold value of 1.5 provided $63.1 \%$ sensitivity and $69.2 \%$ specificity. In addition, we divided the SFA calcification into three levels (Fig. 3): no SFA calcification (score 0); mild SFA calcification (score 1-4); and severe SFA calcification (score 5-8). As shown in Fig. 4, with the severity SFA calcification elevated, the prevalence of diabetic microvascular complications was significantly increased [diabetic neuropathy: $73(49.0 \%)$ vs. $70(70.0 \%)$ vs. 84 (76.3\%); diabetic nephropathy: 27 (18.1\%) vs. 28 (28.0\%) vs. 50 (45.5\%); diabetic retinopathy: $22(14.8 \%)$ vs. $33(33.0 \%)$ vs. 48 (43.6\%); all $P<0.05]$.

\section{Characteristics of T2DM Patients According to Different SFA Calcification Severity}

Then, we compared the characteristics among T2DM patients with different SFA calcification severity. As shown 
Fig. 3 Representative ultrasound images of 3 levels of SFA calcification in T2DM patients. No SFA calcification (score 0 ): score 0 in the left SFA (1L) and score 0 in the right SFA (1R) from a 52-year-old man; Mild SFA calcification (score 2): score 1 in the left SFA (1L) and score 1 in the right SFA (1R) from a 54-year-old woman. Severe SFA calcification (score 8): score 4 in the left SFA (1L) and score 4 in the right SFA (1R) from a 64-year-old man. The white arrows indicate MAC. MAC medial arterial calcification, $S F A$ superficial femoral arteries, T2DM type 2 diabetes
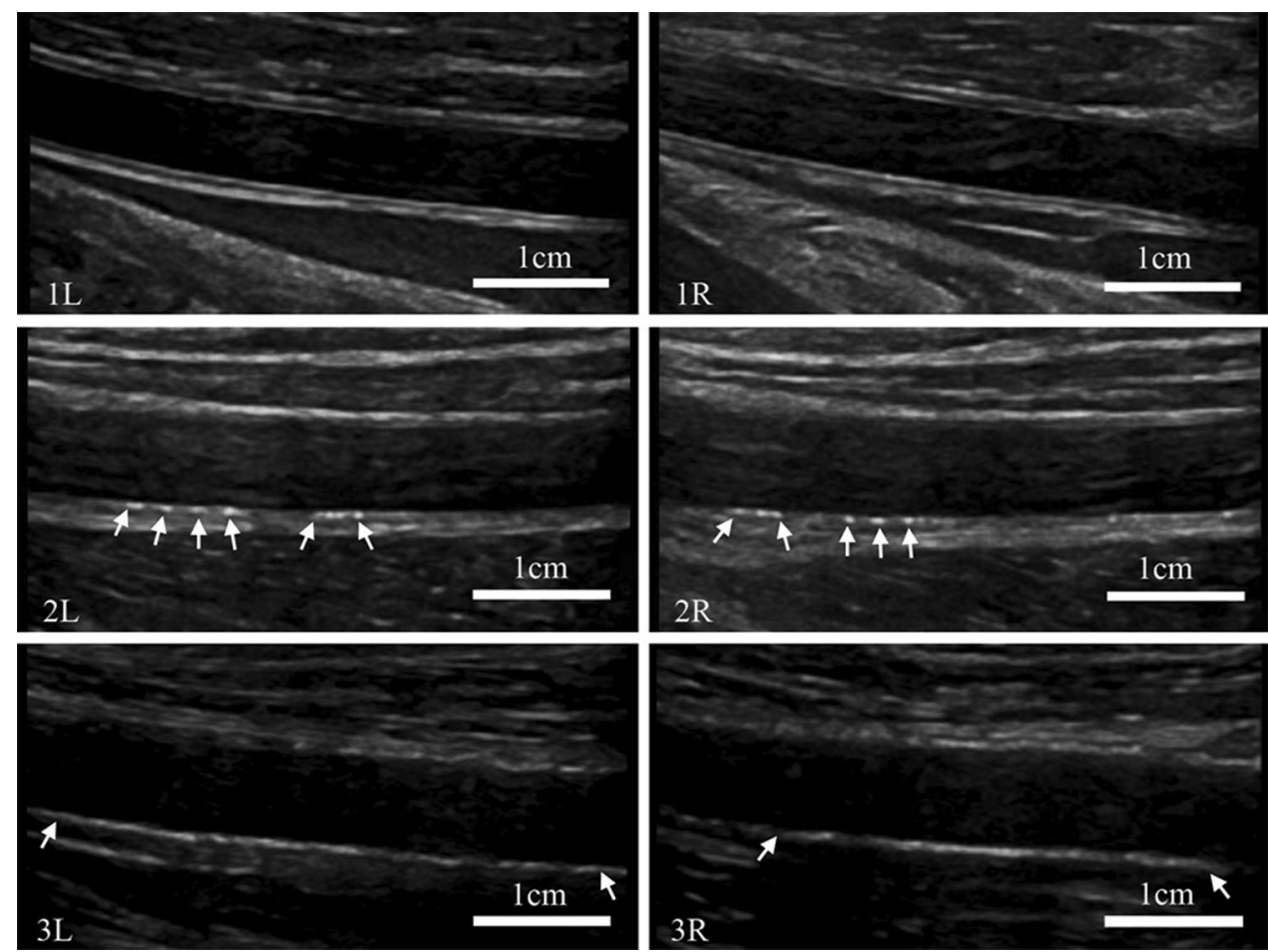

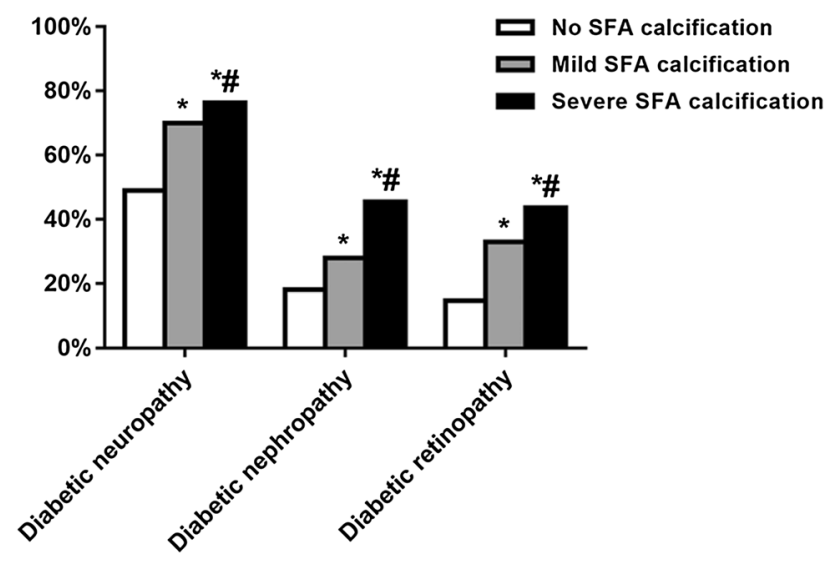

Fig. 4 The prevalence of T2DM-related microvascular complications among different SFA calcification severity groups. SFA superficial femoral arteries, $T 2 D M$ type 2 diabetes. ${ }^{*} P<0.05$ vs. no SFA calcification group; ${ }^{\#} P<0.05$ vs. mild SFA calcification group

in Table 2, age, duration of T2DM, and SBP were significantly elevated with the severity of SFA calcification increased $[53.0 \pm 10.9$ vs. $61.2 \pm 9.3$ vs. $63.8 \pm 11.3$ years; $0(0,8.0)$ vs. $6.0(0,12.8)$ vs.10.0 $(4.0,15.3)$ years; $129.7 \pm 17.7$ vs. $136.2 \pm 22.3$ vs. $140.6 \pm 23.4 \mathrm{mmHg}$; all $P<0.05]$. As regard to the biomedical characteristics, T2DM patients with severe SFA calcification had significantly lower FPG, TyG index and eGFR than those with mild or no SFA calcification $[7.8 \pm 2.6$ vs. $8.8 \pm 3.9$ vs. $9.1 \pm 3.4 \mathrm{mmol} / \mathrm{L}$; $9.16 \pm 0.73$ vs. $9.22 \pm 0.77$ vs. $9.39 \pm 0.74 ; 64.77 \pm 14.14$ vs. $76.10 \pm 12.55$ vs. $83.81 \pm 14.89 \mathrm{~mL} / \mathrm{min}$ per $1.73 \mathrm{~m}^{2}$; all $P<0.05]$. There were no significant differences of sex, smoking, SBP, DBP, BMI, WC, serum lipids, calcium and phosphorus among three groups (all $P>0.05$ ).

\section{Reduced eGFR Is an Independent Risk Factor of SFA Calcification}

We further performed logistic analysis to explore the potential risk factors of SFA calcification. On the univariate logistic analysis, age (OR 1.090; 95\% CI 1.064-1.117; $P<0.001$ ), duration of T2DM (OR 1.090; 95\% CI 1.064-1.117; $P<0.001$ ), SBP (OR 1.021; 95\% CI 1.010-1.032; $P<0.001$ ), TyG index (OR 0.688; 95\% CI $0.517-0.917$; $P=0.011)$ and eGFR (OR 0.925; 95\% CI 0.905-0.945; $P<0.001)$ were significantly associated with the presence of SFA calcification. However, when we put these factors into the multivariate logistic analysis, only age (OR 1.053; 95\% CI 1.025-1.082; $P<0.001)$ and eGFR (OR 0.953; 95\% CI $0.931-0.976 ; P<0.001)$ were independent risk factors of SFA calcification (Table 3 ).

\section{Identifying the T2DM Patients with High Risk of SFA Calcification}

Since aging is an unmodifiable fact OR we then further investigated the association between eGFR and SFA stratified by traditional cardiovascular risk factors, including age, sex, smoking, SBP, BMI and HbA1c, respectively. As shown 
Table 2 Characteristics of T2DM patients among different SFA calcification severity groups

\begin{tabular}{|c|c|c|c|c|}
\hline Variables & $\begin{array}{l}\text { No SFA calcification } \\
N=149\end{array}$ & $\begin{array}{l}\text { Mild SFA calcification } \\
N=100\end{array}$ & $\begin{array}{l}\text { Severe SFA calcification } \\
N=110\end{array}$ & $P$ values \\
\hline Age (years) & $53.0 \pm 10.9$ & $61.2 \pm 9.3^{*}$ & $63.8 \pm 11.3^{*}$ & $<0.001$ \\
\hline $\operatorname{Sex}(M / F)$ & $60 / 89$ & $43 / 57$ & $59 / 51$ & 0.090 \\
\hline Smoking $[N(\%)]$ & $54(36.2)$ & $36(36.0)$ & $27(24.5)$ & 0.097 \\
\hline Duration of T2DM (years) & $0(0,8.0)$ & $6.0(0,12.8)^{*}$ & $10.0(4.0,15.3)^{* \#}$ & $<0.001$ \\
\hline $\mathrm{SBP}(\mathrm{mmHg})$ & $129.7 \pm 17.7$ & $136.2 \pm 22.3 *$ & $140.6 \pm 23.4^{*}$ & $<0.001$ \\
\hline DBP (mmHg) & $79.1 \pm 10.8$ & $77.5 \pm 13.2$ & $78.5 \pm 11.2$ & 0.550 \\
\hline $\operatorname{BMI}\left(\mathrm{g} / \mathrm{m}^{2}\right)$ & $24.3 \pm 3.3$ & $23.9 \pm 2.8$ & $23.6 \pm 3.2$ & 0.183 \\
\hline $\mathrm{WC}(\mathrm{cm})$ & $88.7 \pm 8.5$ & $88.1 \pm 6.8$ & $88.5 \pm 10.7$ & 0.885 \\
\hline FPG (mmol/L) & $9.1 \pm 3.4$ & $8.8 \pm 3.9$ & $7.8 \pm 2.6^{* \#}$ & 0.006 \\
\hline $\mathrm{HbAc1}(\%)$ & $9.7 \pm 2.5$ & $9.5 \pm 2.8$ & $9.4 \pm 2.6$ & 0.722 \\
\hline $\mathrm{TG}(\mathrm{mmol} / \mathrm{L})$ & $1.65(1.21,2.36)$ & $1.51(1.01,2.34)$ & $1.42(1.09,2.11)$ & 0.262 \\
\hline $\mathrm{TC}(\mathrm{mmol} / \mathrm{L})$ & $5.12 \pm 1.73$ & $5.01 \pm 1.36$ & $4.78 \pm 1.56$ & 0.231 \\
\hline HDL-C (mmol/L) & $1.11 \pm 0.39$ & $1.08 \pm 0.28$ & $1.07 \pm 0.29$ & 0.526 \\
\hline $\mathrm{LDL}-\mathrm{C}(\mathrm{mmol} / \mathrm{L})$ & $3.20 \pm 1.02$ & $3.20 \pm 0.97$ & $3.00 \pm 1.08$ & 0.230 \\
\hline Calcium (mmol/L) & $2.25 \pm 0.12$ & $2.24 \pm 0.12$ & $2.22 \pm 0.13$ & 0.160 \\
\hline Phosphorus (mmol/L) & $1.19 \pm 0.21$ & $1.17 \pm 0.19$ & $1.18 \pm 0.21$ & 0.620 \\
\hline TyG index & $9.39 \pm 0.74$ & $9.22 \pm 0.77$ & $9.16 \pm 0.73^{* \#}$ & 0.029 \\
\hline eGFR $\left(\mathrm{mL} / \mathrm{min} \cdot\right.$ per $\left.1.73 \mathrm{~m}^{2}\right)$ & $83.81 \pm 14.89$ & $76.10 \pm 12.55^{*}$ & $64.77 \pm 14.14^{* \#}$ & $<0.001$ \\
\hline Metformin $[N(\%)]$ & $62(41.6)$ & $49(49.0)$ & $44(40.0)$ & 0.371 \\
\hline Other hypoglycemic agents $[N(\%)]$ & $73(49.0)$ & $59(59.0)$ & $58(52.7)$ & 0.300 \\
\hline Insulin $[N(\%)]$ & $51(34.2)$ & $35(35.0)$ & $39(35.4)$ & 0.975 \\
\hline
\end{tabular}

Values presented as mean \pm standard deviation or the median (25-75th quartiles)

$B M I$ body mass index, $D B P$ diastolic blood pressure, $e G F R$ estimated glomerular filtration rate, FPG fasting plasma glucose, HbAlc hemoglobin A1c, $H D L-C$ high-density lipoprotein cholesterol, $L D L-C$ low-density lipoprotein cholesterol, $S B P$ systolic blood pressure, $S F A$ superficial femoral arteries, $T 2 D M$ type 2 diabetes mellitus, $T C$ total cholesterol, $T G$ triglycerides, $T y G$ index triglyceride and glucose index, $W C$ waist circumference

$* P<0.05$ vs. no SFA calcification group and

${ }^{\#} P<0.05$ vs. mild SFA calcification group

Table 3 Logistic analysis for the risk factors of SFA calcification in T2DM

\begin{tabular}{llrll}
\hline Variables & OR $(95 \% \mathrm{CI})^{\mathrm{a}}$ & $P$ value & OR $(95 \% \mathrm{CI})^{\mathrm{b}}$ & $P$ value \\
\hline Age & $1.090(1.064-1.117)$ & $<0.001$ & $1.053(1.025-1.082)$ & $<0.001$ \\
Duration of T2DM & $1.103(1.066-1.141)$ & $<0.001$ & $1.037(0.999-1.077)$ & 0.059 \\
Smoking & $0.754(0.483-1.177)$ & 0.214 & - & - \\
SBP & $1.021(1.010-1.032)$ & $<0.001$ & $1.010(0.998-1.022)$ & 0.106 \\
BMI & $0.943(0.881-1.009)$ & 0.090 & - & - \\
FPG & $0.928(0.871-0.989)$ & 0.021 & $0.983(0.901-1.073)$ & 0.699 \\
HbAc1 (\%) & $0.968(0.797-1.043)$ & 0.434 & - & - \\
TC & $0.912(1.047-1.408)$ & 0.178 & - & - \\
TG & $0.944(0.867-1.028)$ & 0.944 & - & - \\
LDL-C & $0.905(0.738-1.111)$ & 0.342 & - & - \\
TyG index & $0.688(0.517-0.917)$ & 0.011 & $0.863(0.575-1.296)$ & 0.478 \\
eGFR & $0.925(0.905-0.945)$ & $<0.001$ & $0.953(0.931-0.976)$ & $<0.001$ \\
\hline
\end{tabular}

$B M I$ body mass index, $e G F R$ estimated glomerular filtration rate, $F P G$ fasting plasma glucose, $H b A l c$ hemoglobin A1c, $L D L-C$ low-density lipoprotein cholesterol, $S B P$ systolic blood pressure, $S F A$ superficial femoral arteries, $T 2 D M$ type 2 diabetes, $T C$ total cholesterol, $T G$ triglycerides, $T y G$ index triglyceride and glucose index

${ }^{\mathrm{a}}$ Results from univariate logistic analysis

${ }^{\mathrm{b}}$ Results from multivariate logistic analysis 


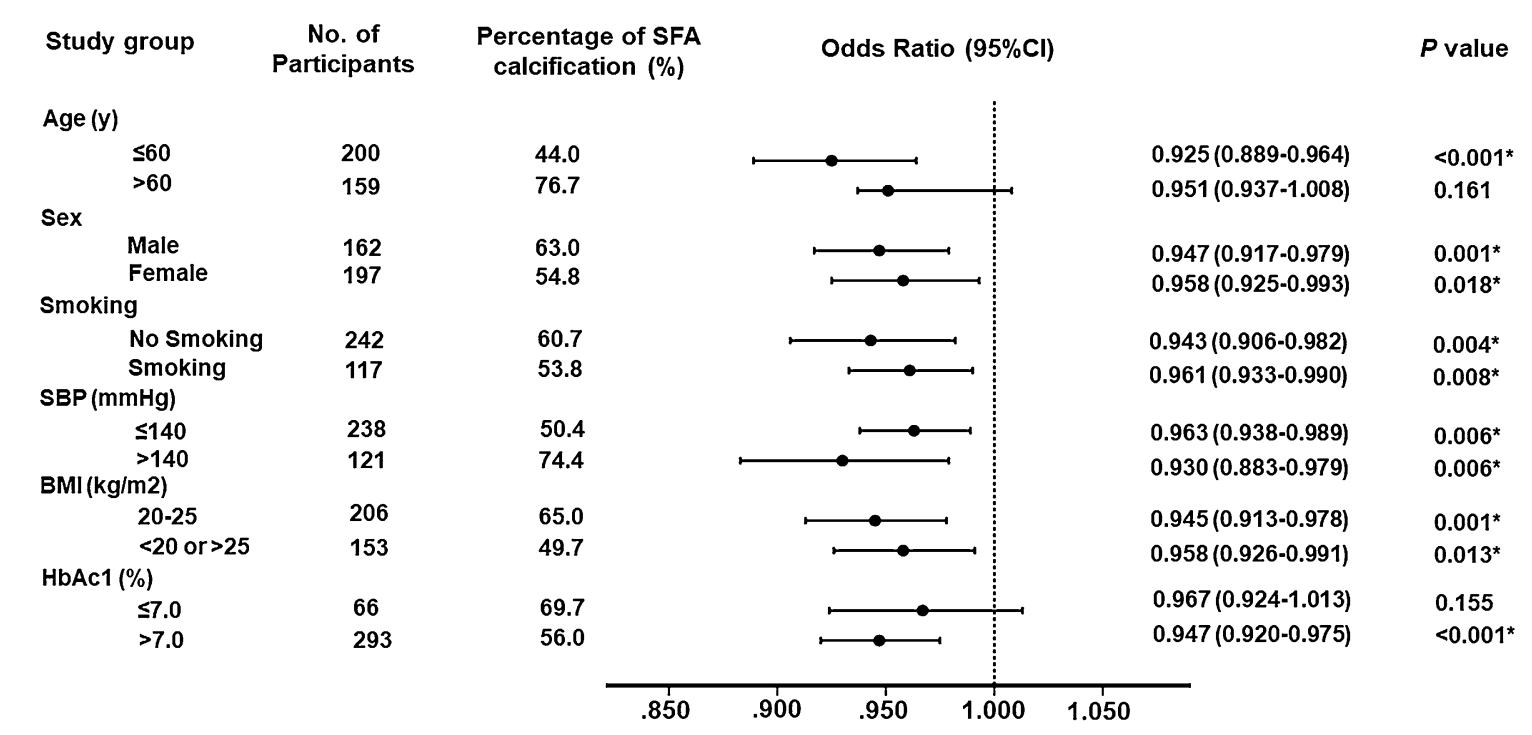

Fig. 5 Subgroup analysis of assessing the association between eGFR and SFA calcification in T2DM. Multivariate logistic analysis after adjustment for age, duration of T2DM, BMI, SBP, and TyG index was performed in subgroup according to age $(\leq 60$ or $>60$ years), sex (male or female), systolic blood pressure (SBP; $\leq 140$

in Fig. 5, we found that the negative association between eGFR and SFA calcification was significant in PA patients with $\leq 60$ years of age (OR 0.925 ; 95\% CI $0.889-0.964$; $P<0.001$ ), HbA1c $>7.0$ (OR 0.947; 95\% CI 0.920-0.975; $P<0.001)$. However, the association between eGFR and SFA calcification became nonsignificant in T2DM patients with $>60$ years of age and $\mathrm{HbA} 1 \mathrm{c} \leq 7.0$ subgroups (all $P>0.05$ ). Moreover, this association remained significant among sex, smoking and BMI subgroups, suggesting it was not influenced by sex, smoking and BMI difference (all $P<0.05)$.

\section{Discussion}

Major findings from our study demonstrated that (1) SFA calcification had a strongest correlation with microvascular complications in patients with T2DM; (2) reduced eGFR was an independent risk factor for SFA calcification, especially in T2DM patients $\leq 60$ years old with $\mathrm{HbA} 1 \mathrm{c}>7.0$.

Microvascular complications are great threats to patients with T2DM, which reduces their life quality and increases the hospitalized duration [22]. MAC is a common condition in T2DM patients, which was shown to associate with various diabetic macrovascular complications [12,13]. Chowdhury et al. showed that CT-based lower limb arterial calcification was strongly associated with cardiovascular events [23]. However, these studies were limited by radiology technique, which was inferior to US in evaluating MAC $[12,13]$. In addition, it is under debate whether below- or above-knee or $>140 \mathrm{mmHg}$ ), and BMI $\left(20-25\right.$ or $\left.>25 \mathrm{~kg} / \mathrm{m}^{2}\right)$. Data were expressed as the odds ratio and $95 \%$ confidence interval (CI). $* P<0.05$. BMI body mass index, $e G F R$ estimated glomerular filtration rate, $S B P$ systolic blood pressure, $S F A$ superficial femoral arteries, $T 2 D M$ type 2 diabetes, $T y G$ index triglyceride and glucose index

calcification plays a role in T2DM-related microvascular complications. Thus, we adapted ultrasonography measurement and compared the roles of MAC in above-knee arteries (SFA), and below-knee arteries (ATA and PTA) in predicting microvascular complications in T2DM. It was emphasized that compared with ATA and PTA, SFA lesion had a more important role in predicting prognosis. McDermott $\mathrm{MM}$ et al. showed that lipid-rich necrotic core in the SFA was associated with higher rates of clinical PAD events [24]. Another study demonstrated that greater plaque quantity and smaller lumen area in the proximal SFA were associated with higher mobility loss in people with PAD [25]. Consistently, our results showed that SFA calcification had the strongest association with various diabetic microvascular complications than ATA and PTA calcification. Thus, early diagnosis and appropriate control of the risk factors of SFA calcification was possibly useful to reduce T2DM-related complications.

Instead of a passive product of calcium and phosphate precipitation, vascular calcification is now regarded as an actively regulated process involving many factors [26]. Although there were a series of studies investigating the risk factors of largersized lumen vascular calcification like coronary artery calcification, few have been known about lower limb arterial calcification in T2DM. Insulin resistance and hyperglycemia are major characteristics of T2DM [27]. It was demonstrated that insulin resistance was associated with coronary artery calcification [28]. As a reliable indicator of insulin resistance, highTyG index was reported to be independently associated with the presence of coronary artery calcification in healthy Korean 
adults [29]. Although our data showed that neither high-TyG index nor FPG was an independent risk factor of SFA calcification, it indicated that under impaired kidney function condition, hyperglycemia may accelerate the development of SFA calcification. It was supported by the fact that the induction of in vitro T2DM calcification model required the high phosphate microenvironment (mimicked the reduced eGFR) [30-32]. Vascular calcification was thought to be a manifestation of vascular aging [33]. We consistently showed that older age was a strong risk factor of SFA calcification. Interestingly, the results of our subgroup analysis suggested that in T2DM patients with bad controlled hyperglycemia, downregulation of eGFR might trigger SFA calcification in the early stage. And this may explain why improving eGFR showed little effect on SFA calcification in older T2DM patients even with better control of blood glucose. However, further investigation is still required to verify our results.

This study had several limitations that should be highlighted. First, the number of T2DM patients enrolled in the study was relatively small. Therefore, further studies with larger sample size are required. Second, because of the cross-sectional design of the present study, causality between reduced eGFR and SFA calcification extent cannot be established. Third, some residual factors may confound our results despite careful adjustment, further studies are needed to verify our findings.

In summary, we provided clinical evidence that SFA calcification was a good predictor of microvascular complications in T2DM patients. Reduced eGFR was probably an important risk factor of SFA calcification in T2DM patients, especially in those young with bad controlled hyperglycemia.

Acknowledgements This work was supported in part by the National Natural Science Foundation of China (NSFC) [81801719] to Xiaolin Xu, NSFC [81900379] to Jingwei Gao and Traditional Chinese Medicine Research Project of traditional Chinese medicine bureau of Guangdong province of China [20191070] to Jing Tian.

Author contributions JT, WBH, JWG designed the study. LY, ML and WYZ contributed to data acquisition. WBH and JWG performed the statistical analysis. JT, JWG, and LY contributed to the discussion. WBH and JWG drafted the manuscript. JT, BML and XLX edited the manuscript. All authors read and approved the final manuscript.

\section{Compliance with Ethical Standards}

Conflict of interest Jing Tian, Wanbing He, Jingwei Gao, Li Yan, Ming Liang, Wenyue Zhang, Xiaolin Xu and Baoming Luo confirms that they have no conflict of interest related to this study.

Human and Animal Rights and Informed Consent This study was approved by the Sun Yat-sen Memorial Hospital of Sun Yat-sen University Research Ethics Committee and all participants provided written informed consent.
Open Access This article is licensed under a Creative Commons Attribution 4.0 International License, which permits use, sharing, adaptation, distribution and reproduction in any medium or format, as long as you give appropriate credit to the original author(s) and the source, provide a link to the Creative Commons licence, and indicate if changes were made. The images or other third party material in this article are included in the article's Creative Commons licence, unless indicated otherwise in a credit line to the material. If material is not included in the article's Creative Commons licence and your intended use is not permitted by statutory regulation or exceeds the permitted use, you will need to obtain permission directly from the copyright holder. To view a copy of this licence, visit http://creativecommons.org/licenses/by/4.0/.

\section{References}

1. Ruta LM, Magliano DJ, Lemesurier R, Taylor HR, Zimmet PZ, Shaw JE (2013) Prevalence of diabetic retinopathy in type 2 diabetes in developing and developed countries. Diabet Med 30:387-398

2. Ilyas Z, Chaiban JT, Krikorian A (2017) Novel insights into the pathophysiology and clinical aspects of diabetic nephropathy. Rev Endocr Metab Disord 18:21-28

3. Tavakoli M, Gogas Yavuz D, Tahrani AA, Selvarajah D, Bowling FL, Fadavi H (2017) Diabetic neuropathy: current status and future prospects. J Diabetes Res 2017:5825971

4. Seuring T, Archangelidi O, Suhrcke M (2015) The economic costs of type 2 diabetes: A global systematic review. Pharmacoeconomics 33:811-831

5. American Diabetes Association (2016) Microvascular complications and foot care. Diabetes Care 39(Suppl 1):S72-S80

6. Valencia WM, Florez H (2017) How to prevent the microvascular complications of type 2 diabetes beyond glucose control. BMJ 356:i6505

7. Lanzer P, Boehm M, Sorribas V, Thiriet M, Janzen J, Zeller T, St Hilaire C, Shanahan C (2014) Medial vascular calcification revisited review and perspectives. Eur Heart J 35:1515-1525

8. Wu M, Rementer C, Giachelli CM (2013) Vascular calcification: an update on mechanisms and challenges in treatment. Calcif Tissue Int 93:365-373

9. Everhart JE, Pettitt DJ, Knowler WC, Rose FA, Bennett PH (1988) Medial arterial calcification and its association with mortality and complications of diabetes. Diabetologia 31:16-23

10. Gujral DM, Shah BN, Chahal NS, Bhattacharyya S, Hooper J, Senior R, Harrington KJ, Nutting CM (2016) Carotid intimamedial thickness as a marker of radiation-induced carotid atherosclerosis. Radiother Oncol 118:323-329

11. Kopel T, Kaufman JS, Hamburg N, Sampalis JS, Vita JA, Dember LM (2017) Endothelium-dependent and -independent vascular function in advanced chronic kidney disease. Clin J Am Soc Nephrol 12:1588-1594

12. David Smith C, Gavin Bilmen J, Iqbal S, Robey S, Pereira M (2008) Medial artery calcification as an indicator of diabetic peripheral vascular disease. Foot Ankle Int 29:185-190

13. Lehto S, Niskanen L, Suhonen M, Rönnemaa T, Laakso M (1996) Medial artery calcification: a neglected harbinger of cardiovascular complications in non-insulin-dependent diabetes mellitus. Arterioscler Thromb Vasc Biol 16:978-983

14. Deas DS Jr, Marshall AP, Bian A, Shintani A, Guzman RJ (2015) Association of cardiovascular and biochemical risk factors with tibial artery calcification. Vasc Med 20:326-331

15. Guzman RJ, Bian A, Shintani A, Stein CM (2013) Association of foot ulcer with tibial artery calcification is independent of peripheral occlusive disease in type 2 diabetes. Diabetes Res Clin Pract 99:281-286 
16. Liu KH, Chu WC, Kong AP, Choi Ko GT, Ma RC, Chan JW, Chow LT, Rasalkar DD, So WY, Tse LF, Corcoran HS, Ozaki R, Ahuja AT, Chan JC (2012) US assessment of medial arterial calcification a sensitive marker of diabetes-related microvascular and macrovascular complications. Radiology 265:294-302

17. Alberti KG, Zimmet PZ (1998) Definition, diagnosis and classification of diabetes mellitus and its complications. Part 1: diagnosis and classification of diabetes mellitus provisional report of a WHO consultation. Diabet Med 15:539-553

18. Vasques AC, Novaes FS, de Oliveira MS, Souza JR, Yamanaka A, Pareja JC, Tambascia MA, Saad MJ, Geloneze B (2011) TyG index performs better than HOMA in a Brazilian population: a hyperglycemic clamp validated study. Diabetes Res Clin Pract 93:e98-e100

19. Tesfaye S, Boulton AJ, Dyck PJ, Freeman R, Horowitz M, Kempler P, Lauria G, Malik RA, Spallone V, Vinik A, Bernardi L, Valensi P; Toronto Diabetic Neuropathy Expert Group (2010) Diabetic neuropathies: update on definitions, diagnostic criteria, estimation of severity, and treatments. Diabetes Care 33:2285-2293

20. Cai W, Li J, Xu JX, Liu Y, Zhang W, Xiao JR, Zhu LY, Liu JY (2184AG) Association of 2184AG polymorphism in the RAGE gene with diabetic nephropathy in Chinese patients with type 2 diabetes. J Diabetes Res 2015:310237

21. Heng LZ, Comyn O, Peto T, Tadros C, Ng E, Sivaprasad S, Hykin PG (2013) Diabetic retinopathy: pathogenesis, clinical grading, management and future developments. Diabet Med 30:640-650

22. Khanam PA, Hoque S, Begum T, Habib SH, Latif ZA (2017) Microvascular complications and their associated risk factors in type 2 diabetes mellitus. Diabetes Metab Syndr 11(Suppl 2):S577-S581

23. Chowdhury MM, Makris GC, Tarkin JM, Joshi FR, Hayes PD, Rudd JHF, Coughlin PA (2017) Lower limb arterial calcification (LLAC) scores in patients with symptomatic peripheral arterial disease are associated with increased cardiac mortality and morbidity. PLoS ONE 12:e0182952

24. McDermott MM, Kramer CM, Tian L, Carr J, Guralnik JM, Polonsky T, Carroll T, Kibbe M, Criqui MH, Ferrucci L, Zhao L, Hippe DS, Wilkins J, Xu D, Liao Y, McCarthy W, Yuan C (2017) Plaque composition in the proximal superficial femoral artery and peripheral artery disease events. JACC Cardiovasc Imaging 10:1003-1012
25. McDermott MM, Carroll T, Carr J, Yuan C, Ferrucci L, Guralnik JM, Kibbe M, Criqui MH, Tian L, Polonsky T, Zhao L, Gao Y, Hippe DS, Xu D, McCarthy W, Kramer CM (2017) Femoral artery plaque characteristics, lower extremity collaterals, and mobility loss in peripheral artery disease. Vasc Med 22:473-481

26. Leopold JA (2015) Vascular calcification: Mechanisms of vascular smooth muscle cell calcification. Trends Cardiovasc Med 25:267-274

27. Laakso M, Kuusisto J (2014) Insulin resistance and hyperglycaemia in cardiovascular disease development. Nat Rev Endocrinol 10:293-302

28. Reaven GM, Knowles JW, Leonard D, Barlow CE, Willis BL, Haskell WL, Maron DJ (2017) Relationship between simple markers of insulin resistance and coronary artery calcification. J Clin Lipidol 11:1007-1012

29. Kim MK, Ahn CW, Kang S, Nam JS, Kim KR, Park JS (2017) Relationship between the triglyceride glucose index and coronary artery calcification in Korean adults. Cardiovasc Diabetol 16:108

30. Heath JM, Sun Y, Yuan K, Bradley WE, Litovsky S, Dell'Italia LJ, Chatham JC, Wu H, Chen Y (2014) Activation of AKT by $\mathrm{O}$-linked $\mathrm{N}$-acetylglucosamine induces vascular calcification in diabetes mellitus. Circ Res 114:1094-1102

31. Lee KM, Lee EO, Lee YR, Joo HK, Park MS, Kim CS, Choi S, Jeong JO, Jeon BH (2018) APE1/Ref-1 inhibits phosphateinduced calcification and osteoblastic phenotype changes in vascular smooth muscle cells. Int J Mol Sci 18:E2053

32. Cazaña-Pérez V, Cidad P, Donate-Correa J, Martín-Núñez E, López-López JR, Pérez-García MT, Giraldez T, Navarro-González JF, Alvarez de la Rosa D (2018) Phenotypic modulation of cultured primary human aortic vascular smooth muscle cells by uremic serum. Front Physiol 9:89

33. Tesauro M, Mauriello A, Rovella V, Annicchiarico-Petruzzelli M, Cardillo C, Melino G, Di Daniele N (2017) Arterial ageing: from endothelial dysfunction to vascular calcification. J Intern Med 281:471-482

Publisher's Note Springer Nature remains neutral with regard to jurisdictional claims in published maps and institutional affiliations. 